\title{
Meniscal Tears: Current Understanding, Diagnosis, and Management
}

Kavyansh Bhan ${ }^{1}$

1. Department of Trauma and Orthopaedics, Whipps Cross University Hospital, London, GBR

Corresponding author: Kavyansh Bhan, kavyanshbhan1993@gmail.com

\begin{abstract}
From once being labelled as a functionless remain of leg muscle, extensive scientific investigations in recent decades have described the meniscus as one of the most crucial structures of the knee. The incidence of meniscal injuries is on the rise and can be attributed to the increased participation of youth in sporting activities. MRI continues to be the imaging modality of choice, and surgical management is the mainstay of treatment for meniscal tears. Arthroscopic partial meniscectomy (APM) is currently the most performed orthopedic procedure around the globe. However, recent studies have conclusively shown that outcomes after an APM are no better than the outcomes after a sham/placebo surgery. Meniscal repair is now being touted as a viable and effective alternative. Meniscal repair aims to achieve meniscal healing while completely avoiding the adverse effects of partial meniscectomy. Meniscal repairs have grown in popularity over the past three decades and have proved to be a much more efficient alternative to partial meniscectomy. It is now increasingly recommended to attempt meniscal repair in all repairable tears, especially in young and physically active patients. Partial Meniscal implants have also shown excellent outcomes in long-term studies, but its efficacy in acute settings still requires further research. Research performed on various techniques of meniscal regeneration looks promising, and regenerative medicine appears to be the way forward. This review aims to critically discuss the current understanding of the meniscus, its role in biomechanics of the knee joint, and the current methods used to diagnose and manage
\end{abstract} meniscal tears.

Categories: Orthopedics

Keywords: meniscus tear, meniscus repair, meniscectomy, regenerative medicine therapies

\section{Introduction And Background}

Though initially described as a functionless remain of a leg muscle [1], extensive scientific investigations in recent decades have described the meniscus as a vital part of the knee joint with anatomical, biomechanical, and functional importance [2]. The incidence of meniscal injuries is on the rise and can be partly attributed to increased participation in sports as well as the recent advances and easy availability of imaging technology such as MRI [3]. A conservative estimate pegs the incidence of meniscal tears at 60 per 100,000, though the true incidence is likely to be grossly underestimated [3]. The literature suggests that knees with known meniscal injury have accelerated cartilage wear, leading to an early onset osteoarthritis. A study by Jarraya et al. found that more than $75 \%$ of patients with symptomatic osteoarthritis have a meniscal injury [4]. In fact, meniscal injury is one of the most common sports injuries in day-to-day practice, and thus its prompt diagnosis and appropriate management have become an increasingly important part of orthopedic research.

Over the last four decades, management of meniscal injuries has seen great advances. Until the 1970s, total meniscectomy was the gold standard in the management of meniscal tears, owing to the then accepted notion of the meniscus being a functionless remnant vestige [1]. However, when postmeniscectomized knees were radiologically examined, it was found the postmeniscectomy knees had femoral condylar flattening, narrowing of joint space, and a predisposition to early degenerative changes [5]. It was suggested that the meniscus has an important weight-bearing function, and its absence interferes with the biomechanics of knee joint, leading to early degenerative changes. This led to the concept of meniscus preservation surgery. Over the years, preservation has shown a high success rate in terms of time to recovery and the functional outcome [6]. This has led to meniscus surgeries being one of the most common orthopedic operations performed, with incidence varying from 17 meniscus procedures per 100,000 population in the United States to 154 procedures per 100,000 in Korea [7]. This review aims to critically discuss the current understanding of the meniscus, its role in biomechanics of the knee joint, and the current methods used to diagnose and manage meniscal tears based on available evidence, and to look into its future potential.

\section{Role of the meniscus}

The meniscus is a relatively avascular structure with extremely limited blood supply. However, it is an indispensable part of biomechanical function of the knee. The meniscus is responsible for increasing the congruence of articulating surfaces of the knee joint [8]. Besides this, meniscus also plays a vital role in shock absorption and load transmission while walking and other activities [9]. Furthermore, it is also helpful 


\section{Cureus}

in providing stability to the knee joint, limiting flexion and extension of the knee joint at extreme angles, and providing proprioception [10]. All these reasons may explain the increasing interest in meniscal injuries over the past few years.

\section{Presentation}

Meniscal tears can cause a range of symptoms, including pain localizing to the joint line, swelling, clicking, catching, locking, and the classic "giving away" of the knee. They are more commonly seen in men as compared to women, with up to $80 \%$ of all meniscal tears being reported in men [9]. Many patients have also reported waking up from sleep due to the pain. This can be explained by the possible scenario of a tender medial aspect of the knee colliding with the other knee while the patient rolls over in his sleep [11]. It is not uncommon to see meniscal injuries in conjunction with damage to structures such as anterior cruciate ligament (ACL), posterior cruciate ligament, or other bony injuries.

\section{Diagnosis}

The onus lies on the orthopedic surgeon to effectively correlate clinical information, radiological images, and his/her clinical expertise to devise an individualized management plan for the meniscal tear. The severity of the symptom seldom corresponds to the type and location of the tear [12]. Detailed historytaking along with thorough clinical examination may not always clinch the diagnosis, and hence radiographic and arthroscopic evaluations should be conducted for confirmation of the diagnosis. Special clinical tests such as McMurray's, Apley's, and Thessaly's tests may have been long recommended for diagnosing a tear, but their accuracy and reliability remain poor [13]. Plain radiographs are not recommended for routine evaluation of meniscus tears and are recommended in select conditions such as chondrocalcinosis [14]. MRI continues to be the imaging modality of choice, with sensitivity and specificity for diagnosing meniscus tears being as high as $93 \%$ and $88 \%$, respectively [15]. On MRI, meniscal tears are usually diagnosed as a linear signal intensity that extends from meniscal substance to a free edge (Figure 1). Diagnostic arthroscopy without a therapeutic component is not recommended.

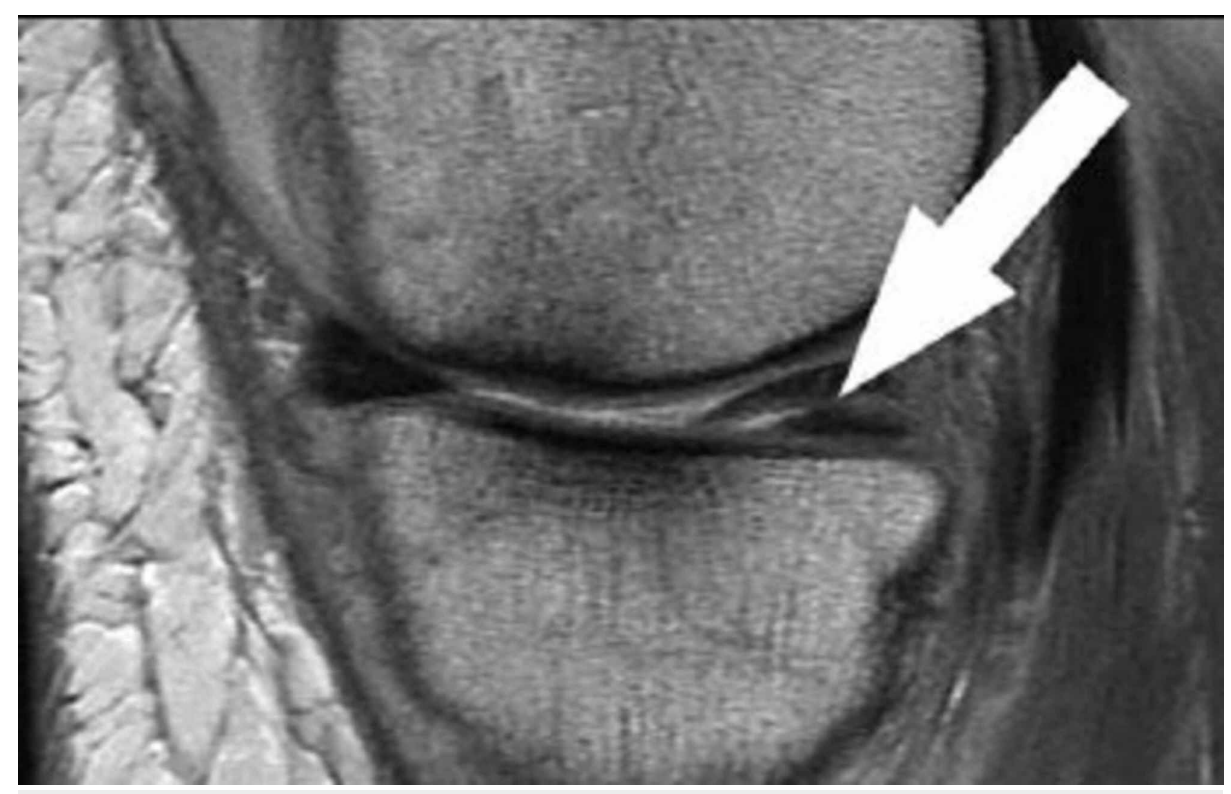

\section{FIGURE 1: An example of a proton-weighted sagittal image showing a posterior horn medial meniscus horizontal tear (white arrow)}

\section{Treatment}

With modern literature emphasizing the vital functions of the meniscus and possibility of early onset osteoarthritis in the absence of the meniscus, orthopedic surgeons have shifted their management goal from resection to preservation, repair, and reconstruction of the meniscus [12]. The treatment, however, still depends on several factors, namely age, comorbidities, symptoms, and type and location of tear. Conservative management is advocated in tears located in the high vascularity zones of the meniscus, namely red/red zone or peripheral $30 \%$ of the medial meniscus and $25 \%$ of the lateral meniscus [16]. Higher success is seen if the said tears measure $<5 \mathrm{~mm}$, as they are considered to be stable tears [16].

Surgical management is the mainstay of treatment for all other types of meniscal tears. Until 1960, open meniscectomy was the standard surgical treatment for meniscal tears [17]. Ikeuchi performed the first arthroscopic repair in 1969, and since then various arthroscopic techniques have evolved [18]. 
Since total meniscectomy can directly lead to a rise in contact stress, an accelerated onset of osteoarthritis, and even symptomatic varus deformities in the older age group, it is now considered to be an almost obsolete treatment option [19]. Arthroscopic partial meniscectomy (APM) is currently the most performed orthopedic procedure around the globe. However, recent studies have conclusively shown that outcomes after an APM are no better than the outcomes after a sham/placebo surgery [20]. It has been termed by many researchers as a "useless" surgery, and recent clinical guidelines have been increasingly recommending against the procedure [21].

Meniscal repair is now being touted as a viable and effective alternative. Meniscal repair aims to achieve meniscal healing while completely avoiding the adverse effects of partial and total meniscectomy. While short-term outcomes of meniscal repairs have been fairly successful, with a failure rate of less than $10 \%$ at two-year follow-ups [22], the long-term results have not been encouraging and failure rates of up to $30 \%$ have been reported at five-year follow-ups [23]. Despite various techniques being developed, such as the inside-out technique, meniscal fixators, all-inside technique, and outside-in technique, failure rates at longterm follow-ups have remained fairly consistent, ranging from $23 \%$ to $30 \%$.

Other treatment options include meniscal allografting. Although a complex procedure, 10-year follow-up survival stood at a promising $89.2 \%$ [24]. Less complex and minimally invasive procedures such as meniscal scaffolds have also been recently approved by the FDA. Scaffolds are available off the shelf and are designed to allow in-growth of tissue on to the scaffold to mimic physiological replacement [25]. Another promising option is a partial meniscal substitute, which is designed to re-establish load distribution across the knee joint, thereby providing a chondroprotective property.

\section{Review}

Over the years, the role of MRI has become indispensable in diagnosing not only meniscal injuries but also almost all the intra-articular pathologies of the knee [26]. Studies have also shown that trained radiologists have a much higher specificity and sensitivity for diagnosing meniscal tears [26]. With clinical examinations having low accuracy in diagnosis, MRI has become the gold standard investigation for meniscal tears. However, owing to its non-invasive nature and high reliability index, MRI is being excessively "medicalized" and being routinely advised in cases where confirmation with an MRI is deemed unnecessary. For instance, patients already diagnosed with severe osteoarthritis on plain X-rays and exhibiting meniscal mechanical symptoms can be safely assumed to have a meniscal tear, and an MRI in such situations does not necessarily change the course of treatment, which would usually be medical management [27].

Surgical management of meniscal injuries has taken rapid strides in recent times. However, the role of the commonest surgery performed worldwide for meniscal tears, APM, remains controversial. Studies have suggested that the rate of cartilage loss can be as high as 7\% per year for healthy young individuals undergoing partial meniscectomy early on in their life [28]. Following an APM, unfavorable outcomes have been reported in as many as $30 \%$ of the cases at a 10-year follow-up [29]. However, due to the easier learning curve and reportedly satisfactory short-term outcomes along with pain reduction at two-year follow-up, APM continues to be one of the most common orthopedic operations being performed worldwide [30].

Over the last two to three decades, great emphasis has been laid on meniscal repair and preservation. The aim is to preserve as much of the meniscus as possible and to avoid meniscectomy. To achieve this, the repair techniques have evolved substantially, with open meniscectomy becoming almost obsolete and arthroscopic techniques being developed consistently. Various arthroscopic techniques such as outside-in, inside-out, and all-inside have been extensively published, with all-inside and inside-out techniques being the most preferred by orthopedic surgeons around the globe [23]. The success of repair, however, depends on a range of factors, most importantly the location and type of the tear. The medial meniscus has its blood supply concentrated in the peripheral $20-30 \%$, whereas the lateral meniscus is perforated by vessels in 10 $25 \%$ of its periphery. The remainder $70-75 \%$ of both menisci receive their nutrition through diffusion in the absence of any direct arterial supply [31]. This has led to the classification of meniscal tears based on their relative location to the blood supply, building on the zonal classification of meniscal tears by Anderson et al. [32]. Tears located at the periphery or zone 1 are referred to as red-red (R/R) tears, tears located in the middle third or zone 2 are referred to as red-white (R/W) tears, and tears located in the inner third or zone 3 are referred to as white-white (W/W) tears [33]. The tears in zones with better blood supply, namely R/R and $\mathrm{R} / \mathrm{W}$, have significantly improved outcomes as opposed to tears in the W/W zone [31]. The morphology of the tears also play an important role in the healing, with horizontal or vertical tears having a success rate of up to $85 \%$ after surgery [34]. The systematic review by Nepple et al. also highlighted the possibility of lateral meniscus tears responding more favorably to repair as opposed to medial meniscus tears [23]. Re-tear rate for medial meniscus tears following a repair has been shown to be as high as $36.4 \%$ [35].

Various studies have compared the outcomes of partial meniscectomy with meniscal repairs. While meniscectomy has better pain relief as opposed to repair in the early stages, it can be attributed to the fact that a meniscectomy removes the pathology immediately by excision of the tear, whereas repairs are based on the principle of regeneration and hence have better outcomes at long-term follow-up [36]. Higher activity levels and better functional outcomes are seen with meniscal repair as opposed to meniscectomy, as it postulated that preservation of the meniscus leads to higher stability of the knees and delays the onset of 
osteoarthritis. Recent studies have shown that repairs exhibit improved healing in knees where ACL is also repaired along with the meniscus [36]. Although no consensus exists on why this happens, various theories have been put forward. Some researchers argue that an ACL reconstruction results in excessive intraarticular trauma to the joint, thereby leading to additional bleeding and fibrin clot formation, which, in turn, means higher availability of growth factors [37]. Another possible explanation is the much slower rehabilitation protocols followed after an ACL reconstruction, thereby producing a low force and more secure environment for meniscal healing [38]. Due to these reasons, meniscal repair is now being encouraged wherever possible.

In conditions wherein repair is no longer a possibility or when there has already been a total or partial meniscectomy performed, meniscus replacement has proved its worth [39]. A replacement may be possible through meniscal allograft transplantation (MAT), whereby the whole meniscus is replaced with or without bone plugs. The risk of disease transmission inherently associated with allografts can be significantly reduced by freezing the graft at -180 degree Celsius with the addition of anti-freezing agents such as glycerol, a technique called cryopreservation [40]. Partial replacement of the meniscus has been achieved by means of meniscal scaffolds. While scaffolds may attempt regeneration of the native meniscus, studies have shown that the tissue grown is different from the native meniscus of the patient [39]. However, a recent systematic review on clinical outcomes of meniscal scaffold reported a failure rate of only $6.7 \%$ to $9.9 \%$ at 45 months' follow-up [41]. An improvement in functional outcomes assessed using Lysholm Knee Score and Tegner Activity Score meant an improvement in the functional activity postmeniscal scaffold replacement [42]. However, despite the promising results, high-quality long-term follow-up studies are required to accurately assess the efficiency of meniscal scaffolds.

A lot of focus has been placed on regenerating the meniscus, and this has propelled further research in regenerative medicine. The use of growth factor for meniscal healing is under development to allow selective control of cell activity in order to promote healing [39]. Another clinical study advocating the use of mesenchymal stem cells in the form of an injection into the knee has shown a considerable increase in the volume of the meniscus at two-year follow-up [43]. Guo et al. have listed the different types of scaffolds currently under development, including absorbable scaffolds, hydrogel scaffolds, and three-dimensional printed scaffolds [44]. These are all exciting concepts that are currently being worked upon.

\section{Conclusions}

Meniscal tears, although a common orthopedic pathology, can be a challenge to treat. Diagnosis of meniscal injuries is not only dependent on good history taking and clinical examination but also almost inevitably requires confirmation with MRI. A thorough understanding of the unique anatomical structure and the knowledge of vascularity along with the zonal classification of tears are extremely important for designing an effective management plan. While conservative management has its role and can be indicated in cases with advanced osteoarthritis or in patients with small tears, partial meniscectomy continues to be the most performed procedure for meniscal injuries. While its efficacy is a matter of debate, its short learning curve and acceptable short-term results have deterred many orthopedic surgeons to switch to more effective procedures. Meniscal repairs have grown in popularity over the past three decades and have proved to be a much more efficient alternative to partial meniscectomy. It is now increasingly recommended to attempt meniscal repair in all repairable tears, especially in young and physically active patients. Partial meniscal implants have also shown excellent outcomes in long-term studies, but their efficacy in acute settings still requires further research. Research performed on various techniques of meniscal regeneration looks promising, and regenerative medicine appears to be the way forward.

\section{Additional Information}

\section{Disclosures}

Conflicts of interest: In compliance with the ICMJE uniform disclosure form, all authors declare the following: Payment/services info: All authors have declared that no financial support was received from any organization for the submitted work. Financial relationships: All authors have declared that they have no financial relationships at present or within the previous three years with any organizations that might have an interest in the submitted work. Other relationships: All authors have declared that there are no other relationships or activities that could appear to have influenced the submitted work.

\section{References}

1. Sutton B: Ligaments: their nature and morphology . Bristol Med Chir J (1883). 1897, 15:344. https://www.ncbi.nlm.nih.gov/pmc/articles/PMC5050733/

2. Makris EA, Hadidi P, Athanasiou KA: The knee meniscus: structure-function, pathophysiology, current repair techniques, and prospects for regeneration. Biomaterials. 2011, 32:7411-31. 10.1016/j.biomaterials.2011.06.037

3. Chambers HG, Chambers RC: The natural history of meniscus tears . J Pediatr Orthop. 2019, 39:53-5. 10.1097/BPO.0000000000001386

4. Jarraya M, Roemer FW, Englund M, et al.: Meniscus morphology: does tear type matter? A narrative review with focus on relevance for osteoarthritis research. Semin Arthritis Rheum. 2017, 46:552-61.

10.1016/j.semarthrit.2016.11.005

5. Fairbank TJ: Knee joint changes after meniscectomy. J Bone Joint Surg Br. 1948, 30:664-70. 10.1302/0301- 
6. Beaufils P, Pujol N: Management of traumatic meniscal tear and degenerative meniscal lesions. Save the meniscus. Orthop Traumatol Surg Res. 2017, 103:237-44. 10.1016/j.otsr.2017.08.003

7. Park JW: Higher meniscus surgery incidence in Korea compared to Japan or the USA . J Korean Med Sci. 2019, 34:233. 10.3346/jkms.2019.34.e233

8. Matar HE, Duckett SP, Raut V: Degenerative meniscal tears of the knee: evaluation and management . $\mathrm{Br} \mathrm{J}$ Hosp Med (Lond). 2019, 2:46-50. 10.12968/hmed.2019.80.1.46

9. Englund M, Guermazi A, Lohmander SL: The role of the meniscus in knee osteoarthritis: a cause or consequence?. Radiol Clin North Am. 2009, 47:703-12. 10.1016/j.rcl.2009.03.003

10. Greis PE, Bardana DD, Holmstrom MC, Burks RT: Meniscal injury: I. Basic science and evaluation . J Am Acad Orthop Surg. 2002, 10:168-76. 10.5435/00124635-200205000-00003

11. Shiraev T, Anderson SE, Hope N: Meniscal tear - presentation, diagnosis and management . Aust Fam Physician. 2012, 41:182-7.

12. Doral MN, Bilge O, Huri G, Turhan E, Verdonk R: Modern treatment of meniscal tears . EFORT Open Rev. 2018, $21: 260-8.10 .1302 / 2058-5241.3 .170067$

13. Smith BE, Thacker D, Crewesmith A, Hall M: Special tests for assessing meniscal tears within the knee: a systematic review and meta-analysis. Evid Based Med. 2015, 20:88-97. 10.1136/ebmed-2014-110160

14. Huysse WC, Verstraete KL, Verdonk PC, Verdonk R: Meniscus imaging. Semin Musculoskelet Radiol. 2008, 12:318-33. 10.1055/s-0028-1100639

15. Maffulli N, Longo UG, Campi S, Denaro V: Meniscal tears. Open Access J Sports Med. 2010, 26:45-54. 10.2147/oajsm.s7753

16. Giuliani JR, Burns TC, Svoboda SJ, Cameron KL, Owens BD: Treatment of meniscal injuries in young athletes. J Knee Surg. 2011, 24:93-100. 10.1055/s-0031-1280877

17. Karia M, Ghaly Y, Al-Hadithy N, Mordecai S, Gupte C: Current concepts in the techniques, indications and outcomes of meniscal repairs. Eur J Orthop Surg Traumatol. 2019, 29:509-20. 10.1007/s00590-018-2317-5

18. Doral MN, Bilge O, Huri G, Turhan E, Verdonk R: Modern treatment of meniscal tears . EFORT Open Rev. 2018, 3:260-8. 10.1302/2058-5241.3.170067

19. Allen PR, Denham RA, Swan AV: Late degenerative changes after meniscectomy. Factors affecting the knee after operation. J Bone Joint Surg Br. 1984, 66:666-71. 10.1302/0301-620X.66B5.6548755

20. Sihvonen R, Paavola M, Malmivaara A, et al.: Arthroscopic partial meniscectomy versus placebo surgery for a degenerative meniscus tear: a 2-year follow-up of the randomised controlled trial. Ann Rheum Dis. 2018, 77:188-95. 10.1136/annrheumdis-2017-211172

21. Roos EM, Thorlund JB: It is time to stop meniscectomy. Br J Sports Med. 2017, 51:490-1. 10.1136/bjsports2016-097273

22. Lee GP, Diduch DR: Deteriorating outcomes after meniscal repair using the Meniscus Arrow in knees undergoing concurrent anterior cruciate ligament reconstruction: increased failure rate with long-term follow-up. Am J Sports Med. 2005, 33:1138-41. 10.1177/0363546505275348

23. Nepple JJ, Dunn WR, Wright RW: Meniscal repair outcomes at greater than five years: a systematic literature review and meta-analysis. J Bone Joint Surg Am. 2012, 19:2222-7. 10.2106/JBJS.K.01584

24. Bin SI, Nha KW, Cheong JY, Shin YS: Midterm and long-term results of medial versus lateral meniscal allograft transplantation: a meta-analysis. Am J Sports Med. 2018, 46:1243-50. 10.1177/0363546517709777

25. Kurzweil PR, Cannon WD, DeHaven KE: Meniscus repair and replacement. Sports Med Arthrosc Rev. 2018, 26:160-4. 10.1097/JSA.0000000000000224

26. Felli L, Garlaschi G, Muda A, Tagliafico A, Formica M, Zanirato A, Alessio-Mazzola M: Comparison of clinical, MRI and arthroscopic assessments of chronic ACL injuries, meniscal tears and cartilage defects. Musculoskelet Surg. 2016, 100:231-8. 10.1007/s12306-016-0427-y

27. Kemp MA, Lang K, Dahill M, Williams JL: Investigating meniscal symptoms in patients with knee osteoarthritis--is MRI an unnecessary investigation?. Knee. 2011, 18:252-3. 10.1016/j.knee.2010.05.011

28. Cicuttini FM, Forbes A, Yuanyuan W, Rush G, Stuckey SL: Rate of knee cartilage loss after partial meniscectomy. J Rheumatol. 2002, 29:1954-6.

29. Lee CR, Bin SI, Kim JM, Lee BS, Kim NK: Arthroscopic partial meniscectomy in young patients with symptomatic discoid lateral meniscus: an average 10-year follow-up study. Arch Orthop Trauma Surg. 2018, 138:369-76. 10.1007/s00402-017-2853-1

30. Feeley BT, Lau BC: Biomechanics and clinical outcomes of partial meniscectomy . J Am Acad Orthop Surg. 2018, 15:853-63. 10.5435/JAAOS-D-17-00256

31. Cinque ME, DePhillipo NN, Moatshe G, Chahla J, Kennedy MI, Dornan GJ, LaPrade RF: Clinical outcomes of inside-out meniscal repair according to anatomic zone of the meniscal tear. Orthop J Sports Med. 2019, 10:1177/2325967119860806. 10.1177/2325967119860806

32. Anderson AF, Irrgang JJ, Dunn W, et al.: Interobserver reliability of the International Society of Arthroscopy, Knee Surgery and Orthopaedic Sports Medicine (ISAKOS) classification of meniscal tears. Am J Sports Med. 2011, 39:926-32. 10.1177/0363546511400533

33. Barber-Westin SD, Noyes FR: Clinical healing rates of meniscus repairs of tears in the central-third (redwhite) zone. Arthroscopy. 2014, 30:134-46. 10.1016/j.arthro.2013.10.003

34. Mutsaerts EL, van Eck CF, van de Graaf VA, Doornberg JN, van den Bekerom MP: Surgical interventions for meniscal tears: a closer look at the evidence. Arch Orthop Trauma Surg. 2016, 136:361-70. 10.1007/s00402015-2351-2

35. Logan M, Watts M, Owen J, Myers P: Meniscal repair in the elite athlete: results of 45 repairs with a minimum 5-year follow-up. Am J Sports Med. 2009, 37:1131-4. 10.1177/0363546508330138

36. Lee WQ, Gan JZ, Lie DTT: Save the meniscus - clinical outcomes of meniscectomy versus meniscal repair . J Orthop Surg (Hong Kong). 2019, 27:2309499019849813-10. 10.1177/2309499019849813

37. Cannon WD Jr, Vittori JM: The incidence of healing in arthroscopic meniscal repairs in anterior cruciate ligament-reconstructed knees versus stable knees. Am J Sports Med. 1992, 20:176-81. $10.1177 / 036354659202000214$

38. Westermann RW, Wright RW, Spindler KP, Huston LJ; MOON Knee Group, Wolf BR: Meniscal repair with 


\section{Cureus}

concurrent anterior cruciate ligament reconstruction: operative success and patient outcomes at 6-year follow-up. Am J Sports Med. 2014, 42:2184-92. 10.1177/0363546514536022

39. Pereira H, Fatih Cengiz I, Gomes S, et al.: Meniscal allograft transplants and new scaffolding techniques . EFORT Open Rev. 2019, 3:279-95. 10.1302/2058-5241.4.180103

40. Mickiewicz P, Binkowski M, Bursig H, Wróbel Z: Preservation and sterilization methods of the meniscal allografts: literature review. Cell Tissue Bank. 2014, 15:307-17. 10.1007/s10561-013-9396-7

41. Houck DA, Kraeutler MJ, Belk JW, McCarty EC, Bravman JT: Similar clinical outcomes following collagen or polyurethane meniscal scaffold implantation: a systematic review. Knee Surg Sports Traumatol Arthrosc. 2018, 26:2259-69. 10.1007/s00167-018-4838-1

42. Pereira H, Cengiz IF, Vilela C, et al.: Emerging concepts in treating cartilage, osteochondral defects, and osteoarthritis of the knee and ankle. Adv Exp Med Biol. 2018, 1059:25-62. 10.1007/978-3-319-76735-2_2

43. Vangsness CT Jr, Farr J 2nd, Boyd J, Dellaero DT, Mills CR, LeRoux-Williams M: Adult human mesenchymal stem cells delivered via intra-articular injection to the knee following partial medial meniscectomy: a randomized, double-blind, controlled study. J Bone Joint Surg Am. 2014, 15:90-8. 10.2106/JBJS.M.00058

44. Guo W, Liu S, Zhu Y, et al.: Advances and prospects in tissue-engineered meniscal scaffolds for meniscus regeneration. Stem Cells Int. 2015, 2015:517520. 10.1155/2015/517520 